ARTÍCULO ORIGINAL

\title{
LA EVASIÓN TRIBUTARIA Y SU INCIDENCIA EN LA ECONOMÍA DEL ECUADOR, 2010-2014
}

\section{TAX EVASION AND ITS INCIDENCE IN THE ECONOMY OF ECUADOR, 2010-2014}

\begin{abstract}
Yesenia Zamora Cusme
Universidad Nacional Mayor de San Marcos

Doctor en Ciencias Contables y Empresariales - obtenido en la Universidad Nacional Mayor de San Marcos - Lima. Magister en Contabilidad y Auditoría - obtenido en la Universidad Estatal del Sur de Manabí. Economista - obtenido en la Universidad Técnica de Manabí.

Correo: yesymar08@hotmail.com

[Recibido: 07/08/2017 Aceptado: 01/04/2018]

\section{RESUMEN}

El desarrollo del artículo, permitió analizar la evasión tributaria y su incidencia en la economía del ecuador, en el periodo 20102014, el Ecuador cuenta con un sistema tributario fortalecido debido a políticas ejecutadas por el Gobierno de los últimos años, sin embargo existen brechas en la recaudación tributaria, uno de los principales problemas que afectan e impiden este proceso sea efectivo es la evasión de los impuestos por parte de los contribuyentes, siendo este un determinante en los recursos fiscales, el incumplimiento en el pago de impuestos provoca escasez de riquezas y sobre todo se deforma la parte que aborda la economía y sociedad del país. A partir del problema se realizó un estudio de tipos no experimental, bibliográfico, cualitativo, cualitativo, correlacional y transversal de las variables planteadas, obteniendo como principales resultados que las política adoptadas por el Ecuador en base a los tributos y el desconocimiento constante de las normas son causales de evasión, así como también la falta de cultura tributaria, la percepción de que exista bajo riesgo de ser detectado son factores que disminuyen la recaudación de impuestos en el Ecuador, determinante que influye directamente en la economía del país en este periodo.
\end{abstract}

Palabras clave : Evasión Tributaria, Inversión social, Incidencia económica, Riesgo, Recaudación tributaria.

\begin{abstract}
The article analyzes tax evasion and its incidence in the economy of Ecuador, in the period 2010-2014, Ecuador has a strengthened tax system due to policies implemented by the Government in recent years, however there are gaps in tax collection. One of the main problems that affect and prevent this process is effective tax evasion by taxpayers, this being a determinant in tax resources, failure to pay taxes causes scarcity of wealth and -above all-the economic and social structure of the country deteriorates. Based on the problem, a non-experimental, bibliographic, qualitative, qualitative, correlational and cross-sectional study of the variables was carried out. As main results, we obtained that the policies adopted by Ecuador based on taxes and the constant ignorance of the norms are causes of evasion, as well as the lack of tax culture, and the perception that there is a low risk of being detected are factors that reduce the collection of taxes in Ecuador, a factor that directly influences the economy of the country in this period.
\end{abstract}

Keywords : Tax Evasion, Social Investment, Economic incidence, Risk, Tax Collection.

\section{INTRODUCCIÓN}

La investigación relaciona las variables de la evasión tributaria y la incidencia en la economía del Ecuador, para el efecto se formula la siguiente interrogante ¿Cómo analizar el fenómeno de la evasión tributaria $\mathrm{y}$ su efecto en la economía del Ecuador?.La hipótesis que da solución a la interrogante es "En qué medida la reducción de la evasión tributaria podrá impactar a la economía del Ecuador, en el periodo 2010-2014". Para tal efecto, se exponen las teorías de Adam Smith y el contexto histórico y epistemológico de los tributos (Miranda, 2016), las principales teo-

( ) Los autores. Este artículo es publicado por la Revista Quipukamayoc, Universidad Nacional Mayor de San Marcos. Este es un artículo de acceso abierto, distribuido bajo los términos de la Licencia Creative Commons Atribución-NoComercial-Compartirlgual 4.0 Internacional.(http://creativecommons.org/licenses/by-nc-sa/4.0/), que permite el uso no comercial, distribución y reproducción en cualquier medio, siempre que la obra original sea debidamente citadas. 
rías de la evasión (Fernández, 2015), también se fundamenta el diagnóstico de las causas principales de la evasión tributaria (Aguirre, 2013), así como también se describe las bases teóricas de Inversión social (Crespo) y cultura tributaria (Banfi, 2013).

La investigación realiza un enfoque cuantitativo, por que mide la recaudación tributaria en el periodo de análisis y la incidencia que esta ha tenido en la inversión social del País. Así mismo, también se utilizó un enfoque cualitativo para determinar las asperezas que afectan la correcta recaudación tributaria y el peliigro asociado a la evasión que han tenido los contribuyentes, en este mismo enfoque se evidencio las causas principales de la evasión de tributos y como se evidencia el efecto en el desarrollo de la economía del Ecuador.

Miranda (2016) sostiene que "dentro del contexto histórico y epistemológico del tributo éste tuvo sus inicios hace 4000 años antes de Cristo donde relacionaban la palabra tributo equivalente a tribu teniendo como característica principal la reciprocidad, en el siglo I antes de Cristo el tributo era equivalente a cupo siendo el signo de dominación de Roma y sus reinos, en el siglo XVIII después de Cristo el tributo es equivalente a la palabra aporte generado a través de un contrato social mediante la interrelación de un Estado de derecho y los derechos fundamentales de los ciudadanos ".

Asimismo Miranda (2016) afirma que "en nuestra historia la tributación en el imperio incaico tuvo como característica principal la reciprocidad que se tenían unos a otros mediante el intercambiando su producción, fuerza de trabajo y energía humana "

Por otro lado filósofos como Aristóte- les en su obra de justicia social indica que el sistema tributario no es objeto de una exposición sistemática, pero sí de múltiples referencias y alusiones, con lo que es posible, reconstructivamente, hacerse una idea general.

De todos sus elementos, se puede fijar dos componentes fundamentales: los contribuyentes y los impuestos. Los contribuyentes son, primordialmente, los ciudadanos atenienses, el filósofo manifiesta que ya desde la primera democracia, los ciudadanos están censados, distribuidos en clases, según sus rentas (Garcia, 2011).

Valero, Ramirez y Moreno (2014) sostiene que "Platón indica que desde sus orígenes, la obligación de tributar fue producto de la dominación, es decir de la imposición del dominador sobre el dominado, así fuera en su carácter de gobernante, conquistador $o$ simple opresor, siempre que se valiese de la fuerza en cualquiera de sus múltiples formas convencionales e históricas. Advierte que la esencia del problema tributario pues, no únicamente subsiste y se manifiesta plenamente vigente sino desde años históricos.".

Del Busto (2013) indica que "el ser humano es natural y necesariamente social, dado que aisladamente no se basta. La sociedad no puede subsistir sin una regla obligatoria de convivencia que es la Ley y sin una autoridad que la aplique. La vida social crea un bien común que hace posible la realización de las personas individuales que la viven. La creación de este bien común exige la dación de Leyes Tributarias por quienes están facultados para darlas "

Para fundamentar la presente investigación, se tomó en cuenta que la evasión tributaria es una de las causas que viene afectando a todos los países del mundo y en especial a los países con menor desarrollo, con la finalidad de conocer antecedentes sobre este tema, se buscaron otros investigaciones que a continuación se detallan:

Aguirre y Silva (2013) en una de sus tesis realizada titulada evasión tributaria en los comerciantes de abarrotes ubicados en los alrededores del mercado mayorista del distrito de Trujillo - año 2013. Se encuestó y entrevistó a una muestra de 77 empresas comerciales de abarrotes y se obtuvo como resultado "que las causas que se asocian a la evasión tributaria en los Comerciantes del Mercado Mayorista son el deseo de generar mayores ingresos (utilidades), la excesiva carga y complejidad para tributar y finalmente se acogen a regímenes que no les corresponde. Se concluyó que el sistema tributario poco transparente trae como consecuencia, entre otros, cierre temporal de establecimiento, suspensión de licencias, permisos o autorizaciones vigentes otorgadas por entidades del Estado para el desempeño de sus actividades."

Respecto a la teoría de la evasión de Washco (2015) en su base teórica manifiesta "que la evasión constituye toda acción mediante la cual un individuo no cancela los impuestos que las leyes vigentes les exige".

Por otro lado Mendoza (2016) indica que "la evasión de impuestos o evasión fiscal, es un acto ilegal que consiste en ocultar bienes o ingresos con el fin de pagar menos impuestos".

A continuación, se muestra algunas de las teorías de la evasión:

Teoría microeconómica cuyos autores son Becker (1968), Stigler (1970) ,Allingham Srinivasan (1973), Yitzhaki (1974) y Koskela (1983) ,esta teoría se basa en "que hay que centrarse en la actuación individual de las partes que toman decisiones de la cantidad 
a evadir considerando variables como la probabilidad de ser auditado, sanciones y el tipo impositivo".

Teoría de la equidad cuyos autores son Gordon (1989), Cowell (1992) y Tyler y Smith (1998) ,esta teoría se basa en que "es más probable que los individuos cumplan con las reglas si perciben al sistema como justo".

Teoría de la moral fiscal cuyos autores son Baldry (1987), Frey (1997) y Elffers (2000), esta teoría se basa en "que los individuos tienen una motivación interna para pagar o no pagar impuestos”. Esta teoría surge porque ante iguales posibilidades algunos contribuyentes evaden mucho y otros no evaden.

Teoría de la dinámica socia cuyo autor es Torgler (2007) y se basa en que "los contribuyentes interactúan en un juego, en el que su comportamiento se adapta al de los demás contribuyentes".

Respecto a la teoría de la elusión ,para Santiana (2013), la elusión consiste en "evitar o retrasar el pago de determinados impuestos, utilizando para ello mecanismos y estrategias legales, hay elusión cuando el sujeto pasivo evita que surja la obligación tributaria, disminuye su cuantía o pospone su exigibilidad, emplea para tal fin estrategias expresa o implícitamente contenidas en la ley. Agrega el mismo autor que en los países de habla inglesa se usa la expresión "taxavoidance" para indicar la acción de evitar el impuesto sin infringir la ley. La conducta elusiva debe ser previa al hecho gravado si tiene por fin evitar que nazca el impuesto".

Por otro lado, Maldonado y Muñoz, (2011) señala que la elusión consiste "en que el sujeto pasivo evita el pago del impuesto, empleando medios con- templados en la ley o que no contradicen las disposiciones legales, es decir, la utilización de la norma tributaria para reducir el pago de impuestos. La elusión fiscal no constituye violación a la reglamentación tributaria, es compleja de medir y requiere tener cierto conocimiento del comportamiento de los contribuyentes en forma minuciosa, para saber si eluden o no sus obligaciones fiscales “.

Los autores Amoroso, Andino, Carrasco, et al (2015) en su libro titulado la nueva economía en la nueva constitución del Ecuador, establecen que "la elusión de impuestos hace referencia a las conductas del contribuyente que busca evitar el pago de impuestos utilizando para ello maniobras o estrategias permitidas por la misma ley o por los vacíos de esta. La elusión no es estrictamente ilegal puesto que no se está violando ninguna ley, sino que se está aprovechando mediante una interpretación en ocasiones amañada o caprichosa, situación que es permita por una ley ambigua o con vacíos.”

Como sustento de base teórica se establece la definición de la Teoría de la cultura tributaria dada por Villagómez (2011) ,donde expresa "que la cultura tributaria es el proceso de largo plazo que tiene por objetivo elevar el nivel de conciencia cívica respecto del cumplimiento tributario de los ciudadanos", también indica que "es un conjunto de rasgos distintivos de los valores, la actitud y el comportamiento de los integrantes de una sociedad respecto al cumplimiento de sus obligaciones y derechos ciudadanos en materia tributaria”.

Banfi (2013) indica que "la cultura tributaria se entiende como un conjunto de valores, actitudes y conocimientos adquiridos por los miembros de una sociedad respecto a la tributación, con observancia de las leyes vigentes que rigen la conducta del ser humano, como sujeto capaz de ejercer y responder ante las obligaciones tributarias que del mismo devenga, es decir, este comportamiento debe ser visto no tanto como una obligación sino más bien como el deber de coadyuvar en función de resolver las necesidades de un país”.

El objetivo primordial de la presente investigación es la identificación de algunas de las causales incidentes en la evasión de tributos, las misma que pretende mitigar este fenómeno en Ecuador, por medio de este estudio el Servicio de Rentas Internas podría buscar alternativas de solución tomando en cuenta estas dificultades que afectan este proceso, con el fin de lograr reducir el incumplimiento tributario por parte de los contribuyentes y de esta manera mejorar los ingresos provenientes de los tributos.

\section{MATERIAL Y MÉTODOS}

Para el desarrollo de este artículo se realizó la investigación no experimental que permitió observar los hechos tal y como suceden, además se realizó el estudio cualitativo el mismo que consistió en estudiar una parte de la realidad de los contribuyentes en un determinado contexto y el cuantitativo que consistió en la identificación de los valores recaudados y la estimación de los no recaudados por causa de la evasión de tributos.

En la investigación la técnica utilizada ha sido la encuesta, este instrumento permite la elaboración de un cuestionario con preguntas estandarizadas, con la finalidad de obtener información respecto a la gestión tributaria, interés en pago de los impuestos por parte de los contribuyentes seleccionados de la población total. 


\section{RESULTADOS}

Para la presente investigación se estudiaron las siguientes variables, las que permitieron determinar las causas de la evasión tributaria y su incidencia en la economía del país.

$\mathrm{X}=$ Evasión Tributaria vs $\mathrm{Y}=$ Incidencia en la economía ecuatoriana: $\mathrm{X} 1$ : Comportamiento de la recauda- ción tributaria vs Y1: Inversión social; $\mathrm{X} 2$ : Determinación de las dificultades que afectan el proceso de recaudación vs Y2: Riesgo asociado a la inversión; X3: Diagnostico de las causas principales de la evasión vs Y3: El desarrollo de la economía ecuatoriana

Comportamiento de la recaudación tributaria con relación a la inversión. Con la finalidad de caracterizar el comportamiento de los tributos y su incidencia en la inversión social en el periodo 2010-2014., se realizó la investigación de campo, mediante recopilación de información se obtuvo los valores reales recaudados por concepto de tributos en el Ecuador en los periodos de estudios, así como también lo invertido en lo social.

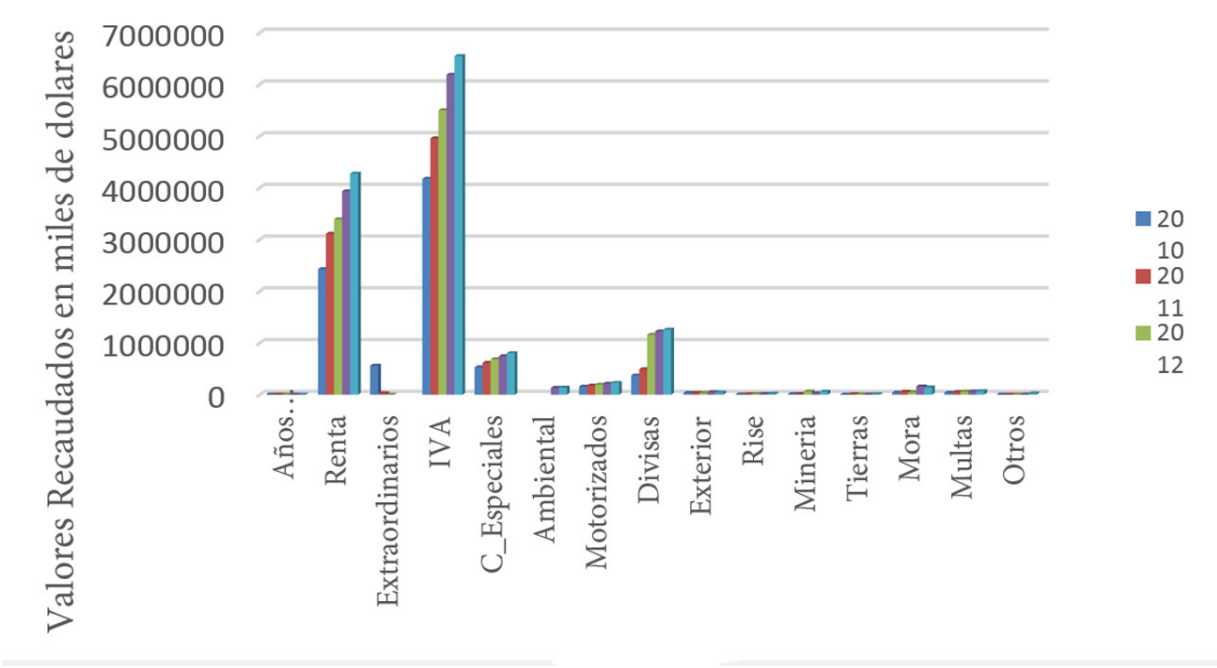

Figura 1. Recaudación de impuestos periodo 2010 -2014.

Fuente: SRI - Elaboración propia.

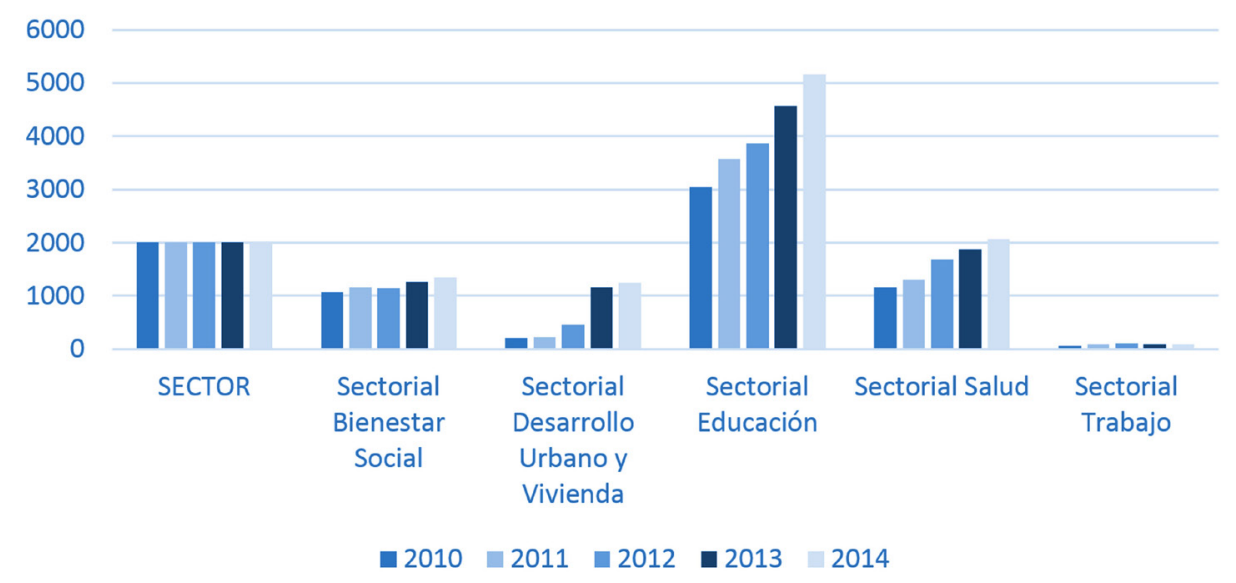

Figura 2. Inversión Social 2010 -2014. Fuente: SRI - Elaboración propia. 
Interpretación (Figura 1 y 2 )

La recaudación de tributos del año 2010 fue del total de efectivo el $109,4 \%$ de la meta presupuestada, el crecimiento nominal del año 2009 comparado con el 2010 fue de 17,50\% del total global, los impuestos recaudados aumentaron progresivamente siendo los directos el $44 \%$ y los indirectos el $56 \%$ del total recaudado, en el año 2011, la recaudación total efectivo supero la meta establecida en el presupuesto general del estado en un $9,8 \%$, si se realiza un análisis comparativo con el año 2010 se observa un crecimiento de la recaudación fue del 14,4\%. Los ingresos por impuestos directos fueron el $42 \%$ y los ingresos por recaudación de tributos indirectos el $68 \%$, el sujeto activo ente regulador de tributos en el Ecuador se plante para el año 2012 como meta por recaudación de total efectivo $\$ 10.560 .000 .000,00$; pero la recaudación dentro de este rubro en el periodo fue de $\$ 11.090 .656 .059,00$ superando así en un $6,7 \%$ la meta establecida. Vale indicar que de los ingresos el $45 \%$ corresponde a im- puestos directos y el 55\% a impuestos indirectos y que los rubros como significancia recaudados son el impuesto al valor agregado e impuesto a la renta, en la comparación de este año con el 2011, se determina que se obtuvo un crecimiento de $17,8 \%$, el cumplimiento de la meta de recaudación del Servicio de Rentas Internas en el año 2013 en el total de efectivo fue del 2,1\% de lo propuesto a recaudar en este periodo, el crecimiento recaudado entre el 2012 y 2013 es el 13,3\% del total efectivo, los ingresos directos correspondieron al $46 \%$ y los indirectos al 54\%. Evidenciando mediante el cuadro \# 04, que los impuestos más significativos en recaudación sigue siendo el impuesto al valor agregado, impuesto a la renta y tenemos un aumento proporcional en el impuesto a la salida de divisas, la recaudación nacional en el año 2014 asciende a $\$ 13616817,193$, superando la meta propuesta en el total de efectivo en un $4 \%$ de lo recaudado, el crecimiento nominal entre los años 2013 - 2014 es del $7 \%$. Todo esto se evidencia en el sector social, el mismo que es primordial por el gobierno nacional, para estos periodos el gasto social ha sido fundamental y el segundo más importante en PGE, siendo de mayor participación los sectoriales de Educación, Salud, Bienestar Social, Desarrollo Urbano, Vivienda y Trabajo.

Determinación de las dificultades que afectan el proceso de recaudación con relación al riesgo asociado a la inversión

Para el cumplimiento de estas variables, se realizó una encuesta diseñada, la que se aplicó a 191 funcionarios de las zonas segmentadas del Servicio de Rentas Internas del Ecuador, para identificar algunas de las dificultades que afectan el proceso de recaudación tributaria en el País. Se realizó un ches list, de 6 preguntas de las falencias más relevantes según lo estudiado en la investigación bibliográfica, la selección se determinó según las opciones que han tenido mayor significancia para los entrevistados.

Se dio un valor a cada pregunta para identificar la pregunta con mayor significancia:

Tabla 1. Dificultades que afectan a la recaudación.

Fuente: Profesionales del Servicio de Rentas Internas - Elaboración propia.

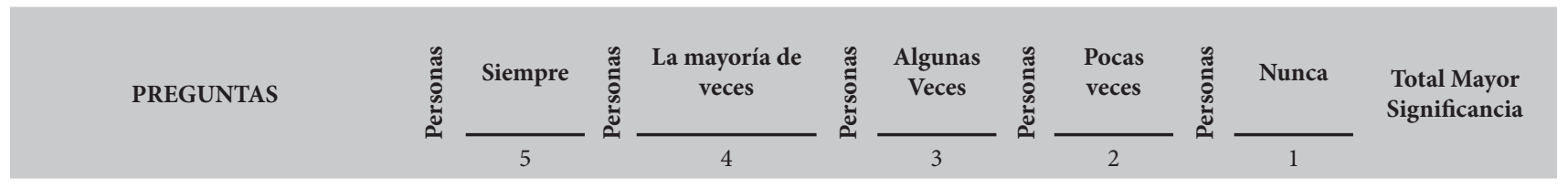

\begin{tabular}{|c|c|c|c|c|c|c|c|c|c|c|c|c|}
\hline \multirow{2}{*}{$\begin{array}{l}1 \\
1.1\end{array}$} & \multicolumn{5}{|l|}{$\begin{array}{l}\text { X2 Dificultades que afectan } \\
\text { la recaudación de impuestos }\end{array}$} & \multicolumn{7}{|c|}{ Respuestas por números de personas } \\
\hline & $\begin{array}{l}\text { Según su criterio la falta de } \\
\text { educación tributaria afecta la } \\
\text { recaudación }\end{array}$ & 105 & 525 & 56 & 224 & 17 & 51 & 8 & 16 & 5 & 5 & 821 \\
\hline 1.2 & $\begin{array}{l}\text { Según su criterio los constan- } \\
\text { tes cambios en la ley y gestión } \\
\text { son dificultades para la recau- } \\
\text { dación }\end{array}$ & 87 & 435 & 37 & 148 & 49 & 147 & 11 & 22 & 7 & 7 & 759 \\
\hline 1.3 & $\begin{array}{l}\text { Según su criterio la falta de } \\
\text { claridad del destino del dine- } \\
\text { ro de los tributos, influye en la } \\
\text { recaudación. }\end{array}$ & 70 & 350 & 23 & 92 & 30 & 90 & 23 & 46 & 45 & 45 & 623 \\
\hline
\end{tabular}




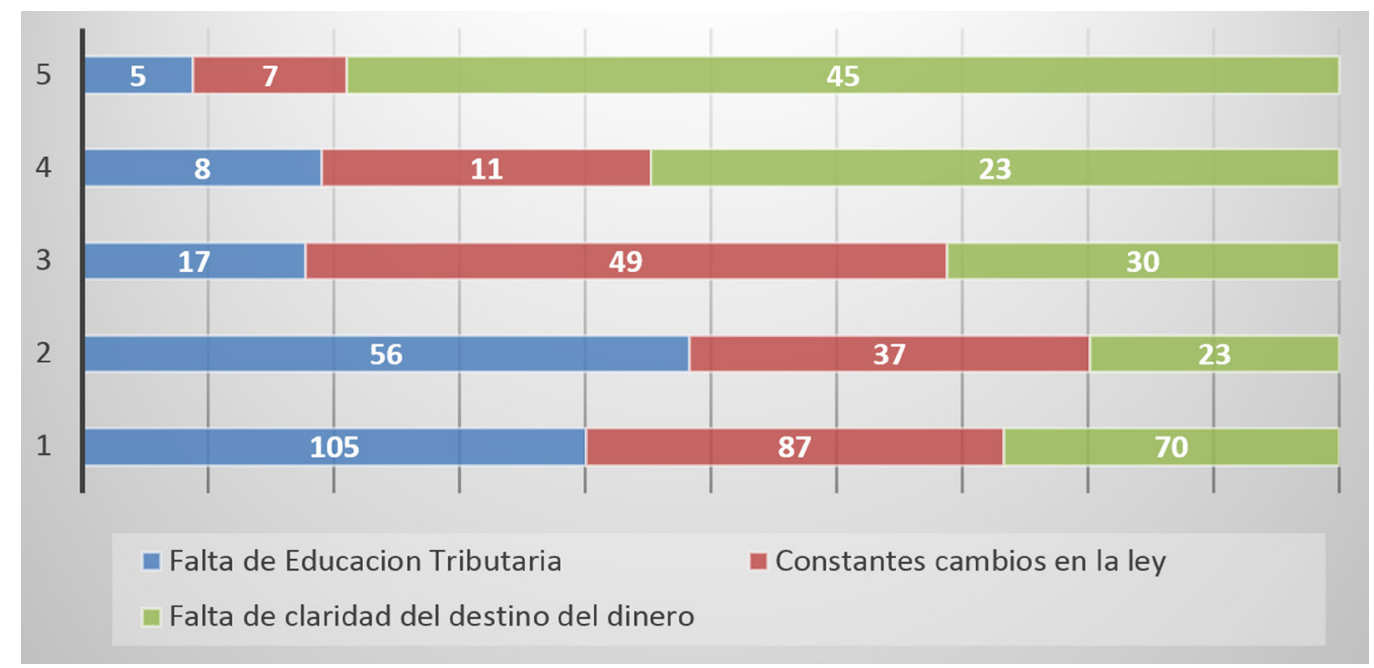

Figura 3. Algunas de las dificultades que afectan la recaudación tributaria.

Fuente: Profesionales del Servicio de Rentas Internas - Elaboración propia.

Interpretación (Figura 3)

La mayoría significancia de las dificultades que afectan la recaudación, según la encuesta realizada a los profesionales que trabajan en el SRI, es la falta de educación tributaria que existe en el país, tomando en cuenta que la administración tributaria trabaja arduamente en este tema.

Es evidente que la falta de educación tributaria siempre va ser un vacío de- terminante en la afectación de la recaudación de tributos, el 54,97\% de los encuestados consideran que este es el factor más relevante de las dificultades cobrar los impuestos o del cumplimento de las obligaciones de los contribuyentes. Los encuestados consideran relevante que los cambios y afectación de la ley son considerables para la afectación de la recaudación, tomando en cuenta que cada modificaron y cambio trae consigo la actualización de los formularios y anexos indispensables para la declaración. El 36,65\% considera que una de las causales de la recaudación de los tributos se da porque no existe una difusión plena de la distribución de estos tributos en el Ecuador, los montos se unifican al presupuesto y no existen líneas específicas que indique que se realiza con cada uno de ellos.

Tabla 2. Riesgo asociado a la evasión tributaria.

Fuente: Profesionales del Servicio de Rentas Internas - Elaboración propia.

\begin{tabular}{|c|c|c|c|c|c|c|c|c|c|c|c|c|}
\hline \multirow{2}{*}{\multicolumn{2}{|c|}{ PREGUNTAS }} & \multirow{2}{*}{ 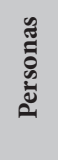 } & Siempre & \multirow{2}{*}{ 胥 } & $\begin{array}{c}\text { La mayoría de } \\
\text { veces }\end{array}$ & \multirow{2}{*}{ 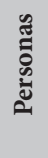 } & $\begin{array}{l}\text { Algunas } \\
\text { Veces }\end{array}$ & \multirow{2}{*}{ 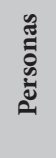 } & $\begin{array}{l}\text { Pocas } \\
\text { veces }\end{array}$ & \multirow{2}{*}{ 胥 } & Nunca & \multirow{2}{*}{$\begin{array}{l}\text { Total Mayor } \\
\text { Significancia }\end{array}$} \\
\hline & & & 5 & & 4 & & 3 & & 2 & & 1 & \\
\hline 2 & Y2: Riesgo Asociado & \multicolumn{11}{|c|}{ Respuestas por números de personas } \\
\hline 1.1 & $\begin{array}{l}\text { Según su criterio las multas e } \\
\text { intereses establecidos en las nor- } \\
\text { mas legales son razonables }\end{array}$ & 20 & 100 & 31 & 128 & 18 & 54 & 31 & 62 & 90 & 90 & 434 \\
\hline 1.2 & $\begin{array}{l}\text { Según su criterio, el cumpli- } \\
\text { miento del pago de tributos los } \\
\text { contribuyentes lo realizan para } \\
\text { evitar ser sancionado }\end{array}$ & 122 & 610 & 23 & 92 & 39 & 117 & 5 & 10 & 2 & 2 & 831 \\
\hline 1.3 & $\begin{array}{l}\text { Según su criterio, el cumpli- } \\
\text { miento en las obligaciones tribu- } \\
\text { tarias los contribuyentes lo rea- } \\
\text { lizan en base al la percepción de } \\
\text { la distribución de los impuestos }\end{array}$ & 110 & 550 & 31 & 124 & 21 & 63 & 17 & 34 & 12 & 12 & 783 \\
\hline
\end{tabular}




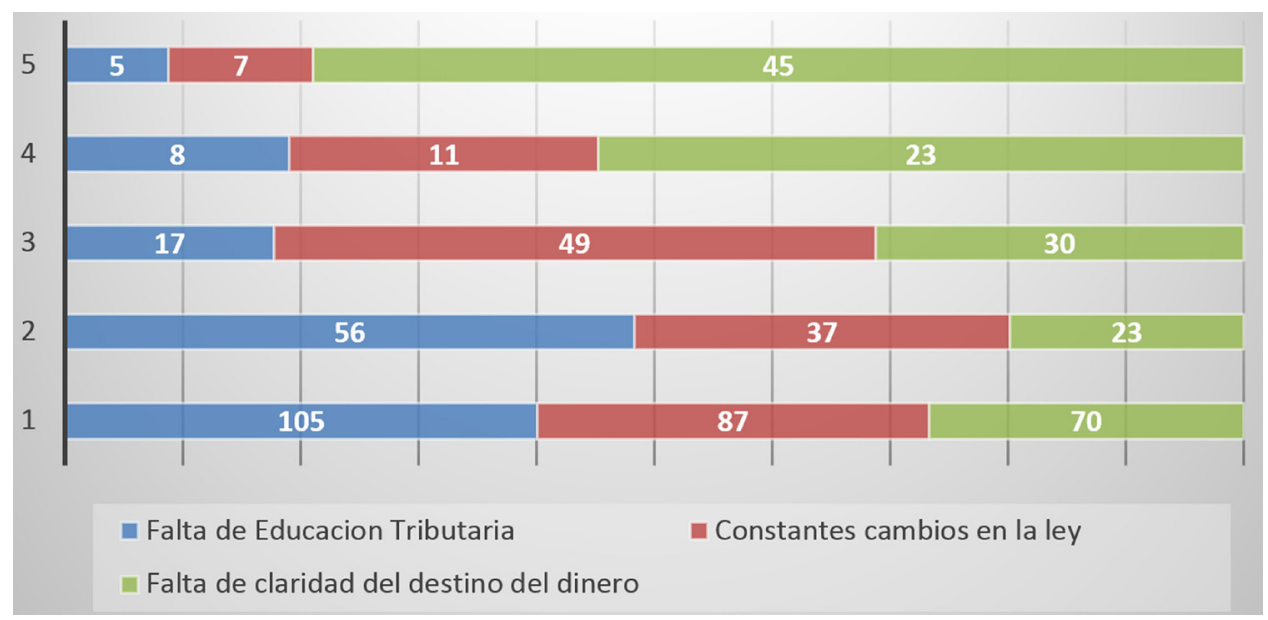

Figura 4. Riesgos asociado a la evasión.

Fuente: Profesionales del Servicio de Rentas Internas - Elaboración propia.

\section{Interpretación (Figura 4)}

La significancia más relevante sobre el riesgo asociado a las dificultades que afectan la evasión de tributos está según la encuesta realizada en el tema asociado a las sanciones, lo que se extiende que los contribuyente cumple más por evitar sanciones que por la contribución con el País.

El 47,12\%; considera que no son razonables las multas e interés establecidos por la administración tributaria, es importante destacar que estos se dan cuando existe el incumpliendo de alguna de las normas establecidas en la ley de Régimen Tributario Vigente en el Ecuador. La mayoría de los contribuyentes realizan los pagos de sus tributar para evitar las sanciones que se dan por el incumplimiento de sus obligaciones, es necesario que se capacita para que los habitantes tributen también en base a la conciencia de mejorar el estado. La percepción que tiene los contribuyentes en la distribución de los impuestos que pagan es relevante en algunas ocasiones para el pago de los tributos, esto también asociado al riesgo que existe evitar el pago de los impuestos.

Causas principales de la evasión con la relación al desarrollo de la economía ecuatoriana

Con la finalidad de evidenciar las causas principales y su incidencia en el desarrollo de la economía ecuatoriana, se realizó la investigación de campo, mediante recopilación de información los siguientes resultados.

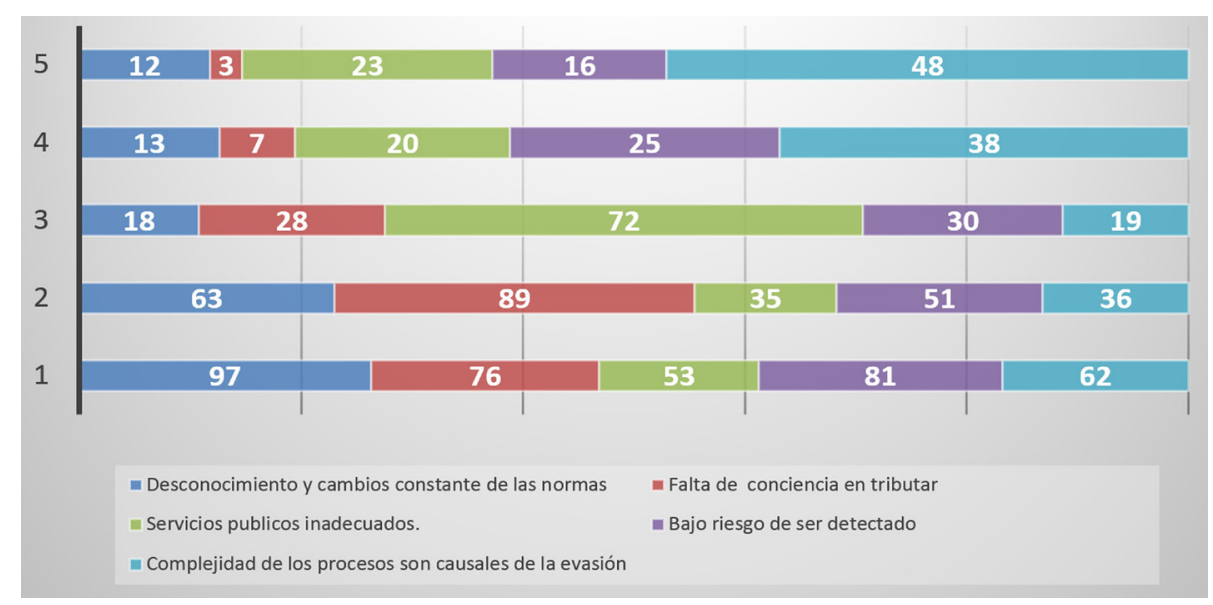

Figura 5. Causas principales de la evasión.

Fuente: Contribuyentes del Servicio de Rentas Internas - Elaboración propia. 


\section{Interpretación (Figura 5)}

Una de las causales más evidentes en la evasión de tributos es el desconocimiento y cambios constante en la ley, el $47,78 \%$, creen que es un determinante para la evasión de tributos. La concientización tributaria que tenga la ciudadanía ecuatoriana en pagar los tributos es un factor indispensable, aunque en la encuesta realizada en un $43,84 \%$, lo consideran un poco relevante, no creen que este sea un factor esencial en la evasión. La percepción de recibir servicios públicos a cambio de los tributos que se pagan no ha sido un factor tan relevante en la encuesta realizada, el 35,47\% considera que algunas veces se considera el servicio público al momento del pago de los impuestos. En esta variable se considera que se encuentra la brecha evasiva más importante en este período, ya que existieron un sinnúmero de contribuyentes que consideran bajo riesgo en la detención y realizaron la evasión, en un programa efectuado por el SRI, se detectaron 514 empresas fantasma que se dedican a la venta de facturas, fueron 7701000 los con- tribuyentes detectados por evasión tributaria, recaudando por conceptos de impuesto a la renta e impuesto al valor agregado 280.793.351 millones de dólares, valores determinados por el servicio de rentas internas. Algunos de los procesos que se realizan para la declaración y presentación de anexos son complejos, se determina que este también es un limitante para la evasión, el 30,54\% de los encuestados consideran que la complejidad de los procesos afecta directamente al incumplimiento de los impuestos.

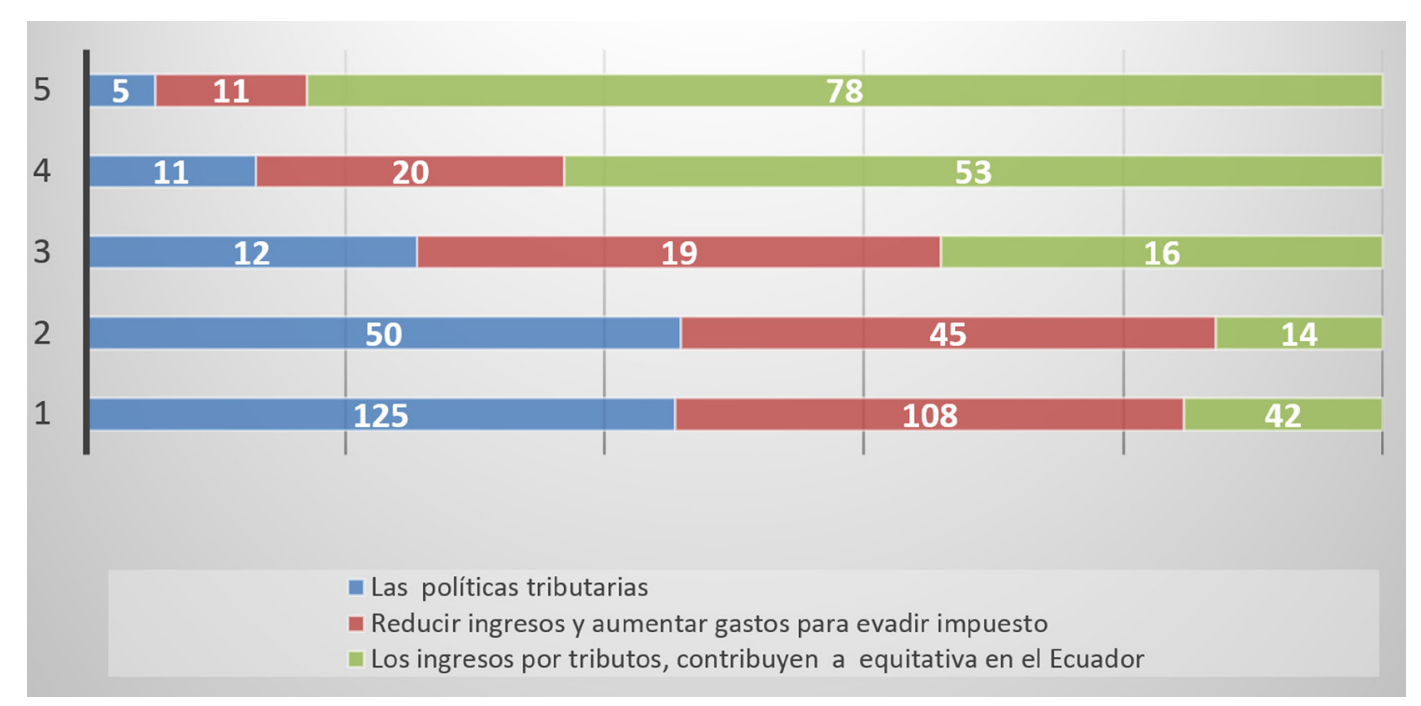

Figura 6. Causas principales de la evasión.

Fuente: Contribuyentes del Servicio de Rentas Internas - Elaboración propia.

\section{Interpretación (Figura 6)}

Si tomamos en cuenta las políticas tributarias adoptadas en el Ecuador y la incidencia que ha tenido en el mejoramiento de la recaudación podríamos decir que disminuye la evasión, pero sin embargo los contribuyentes consideran que entre más leyes o impuestos va existir más evasión, porque las personas siempre van a querer pagar menos. La reducción de ingresos y el aumento de gastos es una causa de evasión determinante, en especial el aumento de gastos irreales que se da con la compra de facturas, que ha sido el determinante más preciso del periodo de estudio de la brecha evasiva determinada por la administración tributaria ente regulador de tributos en el Ecuador. Los contribuyentes conocen el tema y lo considera un factor de la evasión en un 53,20\% de los encuestado lo ha manifestado. Los contribuyentes encuestados consideran que no existe una distribución equitativa y eficiente de los tributos que se pagan, por lo que manifiestan que también esta variable es una de las causas de evasión, ya que el dinero no se destina esencialmente a la inversión social del país.

\section{DISCUSIÓN}

En el análisis realizado al fenómeno de la evasión tributaria estableció que entre las causales más significantes está el cambio constante en las leyes y la gestión, la falta de educación tributaria, el bajo riesgo de ser detectado por la administración, determinante 
que influye directamente en la economía del país en este periodo, ya que encontramos que existieron cantidades de dinero considerable que no se recaudaron en este periodo por causas de estos parámetros, afectando a la economia del Ecuador, ya que se dejó de realizar las obras sociales necesarias.

En la caracterización del comportamiento de los tributos se evidencio que en este periodo la recaudación fue progresiva, los determinantes de este resultado fueron el implemento de nuevos centros de atención, la mejora en la gestión tributaria, las capacitaciones constantes a contribuyentes y las reformas a ley de régimen tributaria que contribuyeron a obtener la eficiente recaudación en el periodo de estudio y esto incidió positivamente en el inversión social ya que se ha mejorado la atención en salud, vivienda, educación, entre otros.

En la determinación de las dificultades que afectan la recaudación de tributos se evidencio que se da por la falta de cultura tributaria, los constantes cambios de la ley y gestión, y la falta claridad del destino de los dineros que se recaudan por concepto de tributos, estableciendo que a pesar de estas dificultades los contribuyentes, tratan de cumplir con sus obligaciones por evitar el riesgo asociados a las sanciones.

El presente estudio determino como causales de la evasión tributaria en el Ecuador el periodo 2010 - 2014, al desconocimiento y cambios constantes de la ley, a la falta de conciencia tributaria, a la percepción de existir bajo riesgo de ser detectado, no percibir servicios adecuados acorde a los montos de tributos que se pagan y en muchas ocasiones a la complejidad de los proceso, lo que ha incidido en la economía del país en estos años ya que no ha permitido una recaudación y distribución equitativa.

\section{REFERENCIAS BIBLIOGRÁFICAS}

Aguirre, A., \& Silva, O. (2013). Eva sion tributaria en los comerciantes de abarrote ubicado en los alrrededores del mercado mayorista del distrito de Trujillo. Trujillo: Universidad Antenor Orrego.

Amoroso, X. \& Carrasco, C. (2015). La Nueva Economía en la Nueva Constitución del Ecuador. Quito; Servicio de Rentas Internas.

Del Busto, J. (2013). Ètica Tributaria. El impuesto. Revista Filosofica y Humanistica, Lima .: 540-546.

Fernandez, C., \& Remache, M. (2015). Evasión en los Impuestos a la Plusvalía y Alcabalas para la Ciudad de Cuenca: análisis a partir de Teoría de Juegos. Cuenca.

Garcia, L. (2011). Fragmentos de una utopía real: Aristóteles y la democracia ateniense. Revista Internacional de Filosofía, 255-256.

Maldonado, M., \& Muñoz, J. (2011). Descripción de las recaudaciones triburtarias en el Ecuador y su relación en los ingresos del estado. Universidad Católica Santiago de Guayaqui, Facultad de Ciencias Económicas, Guayaquil.

Mendoza, A. (2016). Análisis comparativo entre la recaudación del impuesto a la salida de Divisas (Isd) y la del Impuesto a los Consumos Especiales (Ice) En Su Contribución Al Gasto Público, Durante El
Periodo 2008 - 2014. Universidad de Guayaquil, Guayaquil.

Miranda, S. (2016). Influencia de la evasión de impuestos en la recaudación tributaria por las empresas dedicadas a la venta de autopartes im portadas del distrito de la Victoria. Tesis, Lima: Universidad Nacional Mayor de San Marcos, Posgrado

Santiana, A. (2013). Medidas tendientes a combatir la Elusión y lLa Evasión Fiscal de Impuesto a la Renta de las Sociedades en el Ecuador. Quito: Universidad Andina Simón Bolívar.

Silvestri, C., \& Sevilla, K. (2011). Estrategias de aprendizaje de la cultura tributaria en el marco de los estudios a distancia. Caracas.

Valero, M., Ramirez, T., \& Moreno, F. (2014). Etica y Cultura Tributaria en el Contribuyente . Revista Daena: International Journal of Good Conscience, 58-59.

Villagómez, M. (2011). Estudio y análisis de las metas de recaudación del servicio de rentas internas en el ecuador para el período 2005 2009 y su impacto en el presupuesto general del estado. Latacunga: Escuela Politécnica del Ejército,

Washco, T. (2015). Análisis de las reformas tributarias e incidencia en la recaudación de los principales impuestos del Ecuador.Cuenca: Universidad De Cuenca, 